\title{
ROBUST IMAGE TRANSMISSION BASED ON PRODUCT-CODE OPTIMIZATION FOR DETERMINATE STATE LDPC DECODING
}

\author{
N. Thomos ${ }^{1}$, N. V. Boulgouris ${ }^{2}$, and M. G. Strintzis ${ }^{1,3}$ \\ ${ }^{1}$ Informatics and Telematics Institute, Thessaloniki, Greece \\ ${ }^{2}$ Division of Engineering, King's College London, United Kingdom \\ ${ }^{3}$ Electrical and Computer Engineering Department, Aristotle University of Thessaloniki, Greece
}

\begin{abstract}
We propose a novel scheme for error resilient image transmission. The proposed scheme employs a product coder consisting of LDPC codes and RS codes in order to deal effectively with bit errors. The efficiency of the proposed scheme is based on the exploitation of determinate symbols in Tanner graph decoding of LDPC codes and a novel product code optimization technique based on error estimation. Experimental evaluation demonstrates the superiority of the proposed system in comparison to recent state-of-the art techniques for image transmission.
\end{abstract} codes.

Index Terms - Image transmission, Product codes, LDPC

\section{INTRODUCTION}

The widespread deployment of wireline/wireless communication systems and the proliferation of digital media created the recent surge in multimedia transmission research. Since all communication channels suffer from errors, error-resilient schemes have been developed in order to reliably transmit multimedia over unreliable channels. Most of these systems employ embedded scalable source coders, like the SPIHT and the JPEG2000, for image transmission. Unequal Error Protection (UEP) is used for the effective protection of the information and the decoding of satisfactory quality images.

A variety of error-resilient techniques for transmission of images over error-prone channels have been recently proposed in the literature. In [1], irregular repeat accumulate codes (IRA) [2] were proposed for the transmission of JPEG2000 and SPIHT streams over Binary Symmetric Channels (BSC). The scheme in [1] showed performance superior to that of the method in [3]. The transmission of SPIHT streams over BSC channels was also considered in [4]. IRA codes with inherent UEP capabilities were used for reliable communication. The length of the employed IRA codes was equal to the total transmission rate. A product coding scheme based on recursive convolutional codes (RSC) for transmission of SPIHT streams over BSC and Gilbert-Elliot (GE) channels was presented in [5]. This product coder employed a modified Viterbi algorithm (VA) and tried to eliminate transmission errors by using an iterative decoding process.

Email addresses: nthomos@iti.gr, nikolaos.boulgouris@kcl.ac.uk, strintzi@eng.auth.gr. An extended version of this work was submitted to the IEEE Transaction on Image Processing.
In this paper, we propose a novel image transmission methodology based on a product coder consisting of low-density paritycheck (LDPC) codes [6] and Reed-Solomon (RS) codes. The information recovered during each product decoding iteration is considered as a posteriori knowledge and is used for the correction of remaining errors by appropriately modifying the sum product algorithm (SPA). Interleaving is used in order to further improve the efficiency of iterative decoding. A novel UEP approach is proposed which optimizes transmission by calculating error estimates in iterative product decoding. The novel optimization strategy will be shown to yield considerable performance improvements in comparison to other methods for image transmission.

\section{ITERATIVE DECODING OF PRODUCT CODES BASED ON LDPC AND RS}

The proposed product coder is schematically depicted in Fig. 1. It consists of systematic LDPC codes in the horizontal direction and RS codes in the vertical direction. The outer RS codes are used as erasure correcting codes. Due to the progressively decreasing importance of the source information in blocks $1,2, \ldots, k, \ldots, K$ of Fig. 1 the number of RS symbols in the $k_{t h}$ block decreases with increasing $k$. The RS protection is determined using the UEP algorithm presented in Section 4. The inner LDPC codes cope with random errors. The LDPC codes are also used at the decoder in order to indicate packet erasures if the packet bit stream contains uncorrectable errors after the maximum number of LDPC decoding iterations. In the horizontal direction of the product-code array an S-random interleaver is applied in order to randomize errors.

During product decoding, the LDPC decoding of packets corrects bit errors when it is possible, otherwise indicates the erased packers. Then deinterleaving restores the initial arrangement of data in the product-code array. Right after LDPC decoding and deinterleaving, RS decoding takes place in the vertical direction in order to correct the remaining errors. Due to the UEP, the RS decoding will not, in general, correct all errors in a packet, which means that several packets will be only partially recovered. For this reason, we re-decode the LDPC codes in the horizontal direction by taking into account the fact that, due to the preceding RS decoding stage, some bits are deterministically known to be correct $[5,7]$. This additional knowledge boosts the LDPC decoder and enables the correct decoding of some packets that were initially considered erased. This procedure is repeated until no new segment of information is recovered after a product decoding it- 
eration ${ }^{1}$. A simplified block diagram of the above encoding and decoding processes is depicted in Fig. 2. Note that due to the interleaving that is performed after RS decoding in the feedback loop, the LDPC decoding is applied only on corrupted packets in which the determinate bits are evenly distributed.

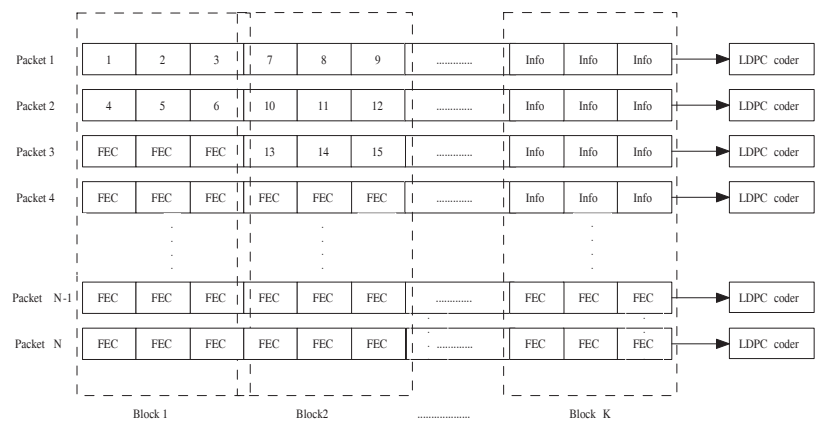

Fig. 1. Product code based on LDPC and Reed-Solomon codes.

\section{EXPLOITATION OF DETERMINATE SYMBOLS IN LDPC DECODING}

In the previous section the iterative decoding of the LDPC/RS product-code scheme was presented. The product coder first attempts to correct all errors using LDPC decoding. Whenever this is not possible, a packet erasure is indicated. The blocks in which the erased packets do not exceed the block's RS protection capability are recovered after RS decoding. Therefore, the portions of the bit stream corresponding to the above blocks are error-free. The recovery of a bit stream portion after a product decoding iteration benefits the subsequent reapplication of LDPC decoding since fewer bits are erroneous and the knowledge that some specific bits are error-free can be used in order to manipulate the soft information exchanged during LDPC decoding iterations.

In order to exploit the deterministic information, we appropriately modified the log-domain version of the SPA, that is usually deployed for LDPC decoding. The Log-Likelihood ratios of the SPA are computed by the following equations:

$$
\begin{gathered}
L\left(c_{i}\right)=\log \left(\frac{\operatorname{Pr}\left(c_{i}=0 \mid \underline{y}\right)}{\operatorname{Pr}\left(c_{i}=1 \mid \underline{y}\right)}\right) \\
L\left(q_{i j}\right)=\log \left(\frac{q_{i j}(0)}{q_{i j}(1)}\right), \quad L\left(r_{j i}\right)=\log \left(\frac{r_{j i}(0)}{r_{j i}(1)}\right)
\end{gathered}
$$

where $c_{i}$ is the bit value at the $i_{t h}$ position of the transmitted codeword, $y$ is the received codeword, $q_{i j}(b)$ is the extrinsic information passed at the first half iteration of LDPC decoding from the variable node $i$ to the check node $j$ and $b \in\{0,1\}$. Respectively, $r_{j i}(b)$ is defined as the extrinsic message passed at the second half of an LDPC iteration from the check node $j$ to the variable node $i$.

Since, in general it is difficult to determine exactly the value of the likelihood ratios we modify the SPA algorithm by biasing the ratios whenever determinate information is available. This means

\footnotetext{
${ }^{1}$ Note that there are two types of iterations. A product decoding iteration involves LDPC decoding and RS decoding, while LDPC iterations are part of LDPC decoding.
}

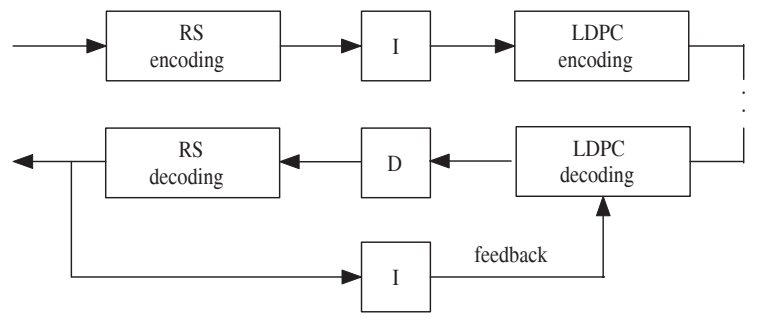

Fig. 2. Encoding and decoding using the proposed scheme. RSdecoded information is feed back to the LDPC decoding module. Interleaving (I) and deinterleaving (D) is performed in order to distribute the determinate bits evenly.

that whenever the correct value of $b$ is known, we enforce the likelihood ratios in eqs. (1) to take the appropriate sign (determined by $b$ ). In practice, we set the likelihood ratio to be equal to a very large positive value, in case $b$ is equal to 0 , or we set it to a very large negative value if $b$ is equal to 1 . This biasing approach is implicity assumed in the analysis that follows.

The decision regarding bit values in LDPC decoding are taken using the log-likelihood ratios $L\left(Q_{i}\right)$, where [6]

$$
L\left(Q_{i}\right)=L\left(c_{i}\right)+\sum_{j \in C_{i}} L\left(r_{j i}\right)
$$

If the transmitted bit at the $i_{t h}$ position $c_{i}$ is determinate and takes the value 1 , then it should be $L\left(Q_{i}\right)<0$. Equivalently, the knowledge that the transmitted bit at the $i_{t h}$ position is 1 means that the following relation should hold

$$
L\left(c_{i}\right)+\sum_{j \in C_{i}} L\left(r_{j i}\right)<0
$$

The term $L\left(c_{i}\right)$ is determined by the channel and the transmitted bit at the $i_{t h}$ position. In an errorless environment, $L\left(c_{i}\right)$ and $L\left(Q_{i}\right)$ should always have the same sign. Thus, whenever $c_{i}$ is determinate, we enforce $L\left(c_{i}\right)$ to have the same sign as $L\left(Q_{i}\right)$. To further improve the decoding performance we set $L\left(r_{j i}\right)<0$ for all $j \in C_{i}$. In a similar manner, we are biasing $L\left(q_{i j}\right)<0$ when $c_{i}=1$. The application of the above approach to the case in which the value of the determinate bit is equal to 0 is trivial. This manipulation of the soft information has a very beneficial impact on the efficiency of LDPC decoding.

\section{NOVEL CHANNEL RATE ALLOCATION BASED ON ERROR ESTIMATES}

The occurrence of determinate bits depend on the initial RS-based UEP that is applied in the product code array. The UEP algorithm that we initially used with the present product coding method was based on dynamic programming and was originally proposed in [7] for the transmission of SPIHT streams over wireless channels. This scheme will be hereafter termed as APC since UEP optimization is based on the a priori channel conditions. The optimization process adjusts the amount of RS protection according to the importance of source information. Therefore, more channel symbols (RS symbols) are allocated to blocks contribute with higher distortion improvement to the eventual image quality and fewer to other 
blocks. In the following, we first briefly present the APC approach and then we focus on the proposed approach for channel rate allocation based on error estimates.

Since RS codes are used, we define the probability $P_{L}(k)$ of losing the $k_{t h}$ block, i.e., the probability that the RS protection, which is allocated to the $k_{t h}$ block is not sufficient to correct the corrupted information in the erased packets. The expected distortion $\bar{D}$ is given by

$$
\begin{aligned}
\bar{D} & =\sigma^{2} \cdot P_{L}(1)+\sum_{k=2}^{K} D_{k-1} \cdot\left(P_{L}(k)-P_{L}(k-1)\right)+ \\
& +\left(1-P_{L}(K)\right) \cdot D_{K}
\end{aligned}
$$

where $D_{k}$ is the distortion after the successful decoding of blocks $1, \ldots, k, K$ is the total number of transmitted blocks and $\sigma^{2}$ is the variance of the pixel intensities. In the following, $k$ is the stage (block) index. The minimization of $\bar{D}$ takes place using dynamic programming.

Although the APC approach will be shown to perform well, it does not take into consideration the iterative product decoding process. Therefore, we would like to optimize the RS rate allocation process, so that the RS decoding provides the optimal number of determinate bits to the LDPC decoder. To this end, we modify the APC technique in order to exploit the determinate bits recovered after a product code iteration. This scheme is referred to as $A E C$ since adaptively-estimated channel conditions are considered during optimizations. The AEC approach differs to APC in that the packet loss probabilities are updated on the fly during optimization, based on the number of available determinate bits (see Fig. 3 ), while in the APC technique the probabilities remain unaltered.

The proposed optimization algorithm is based on dynamic programming. A trellis is constructed in which the number of states is equal to the number of available RS protection levels and the number of stages equals the number of the blocks to be protected (see Fig. 1). Our objective is to minimize the total expected distortion for the transmission of blocks $1, \ldots, K$. To this end, we define the adaptively-calculated probability $\tilde{P}_{L}(k)$ of losing the $k_{t h}$ block when the AEC approach is followed. Specifically,

$$
\begin{gathered}
\tilde{P}_{L}(k)=\operatorname{Prob}\{\text { erased packets }>Q(k)\}= \\
=\sum_{i=Q(k)+1}^{N}\left(\begin{array}{c}
N \\
i
\end{array}\right) \cdot p_{k}^{i} \cdot\left(1-p_{k}\right)^{N-i}
\end{gathered}
$$

where $Q(k)$ denotes the number of RS symbols in each column of the $k_{t h}$ block, $N$ is the number of packets and $p_{k}$ is the packet erasure probability considering that the bits in the first $k-1$ blocks are determinate (see Fig. 3).

Using the above definitions, the optimization algorithm used with AEC can be summarized as follows:

1. Set $k=1$ and $\bar{D}_{0}=\sigma^{2} \cdot \tilde{P}_{L}(1)$.

2. For each trellis node in the $k_{t h}$ stage $^{2}$, i.e., for all possible protection choices for the $k$ th block, compute the expected minimum distortion $\bar{D}_{k}=\bar{D}_{k-1}+D_{k} \cdot\left(\tilde{P}_{L}(k+1)-\right.$ $\left.\tilde{P}_{L}(k)\right)$ and retain only the path associated with the lowest cumulative expected distortion up to that stage.

3. Assume that the bits in the first $k$ blocks are determinate and calculate $\tilde{P}_{L}$ using the estimated erasure probabilities given in Fig. 3.

\footnotetext{
${ }^{2}$ The trellis node index is omitted in our analysis for notational conve-
}

4. Set $k=k+1$. If $k<K$, go to step 2 .

5. $\bar{D}_{K}=\bar{D}_{K-1}+\left(1-\tilde{P}_{L}(K)\right) \cdot D_{K}$

6. Allocate the RS protections $Q(k), k=1, \ldots, K$, that correspond to the path with the overall lowest cumulative expected distortion.

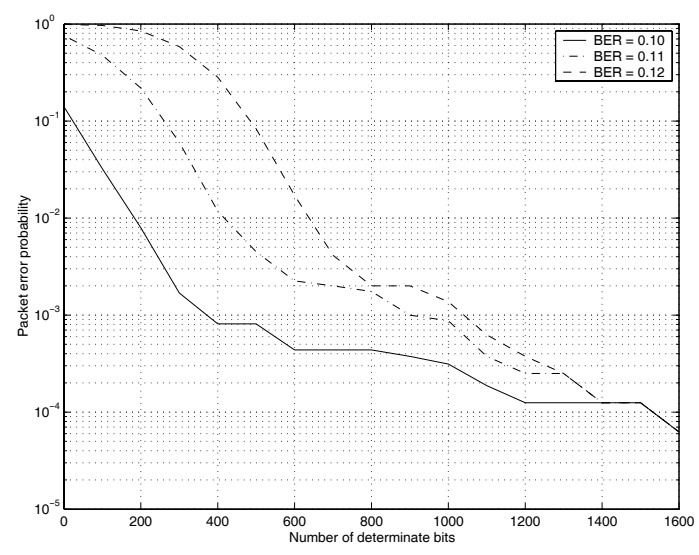

Fig. 3. Packet erasure probabilities of the $(1904,4096)$ LDPC code for variable bit error rates. $50 \mathrm{LDPC}$ iterations were assumed.

\section{EXPERIMENTAL RESULTS}

The proposed schemes were experimentally evaluated for the transmission of SPIHT encoded $512 \times 512$ test image "Lenna" over BSC and Gilbert-Elliot (GE) channels. The considered transmission scenarios were over BSC with BER 0.1 and over the GilbertElliot channels described in [5].

Irregular LDPC codes generated using the algorithm in [8] were employed. Specifically, the $(3880,8272)$ LDPC codes, which employs packets of 1034 bytes, were used with the proposed scheme. The maximum allowable number of LDPC iterations was set to 50. The RS codes utilized by the proposed scheme are defined on $G F\left(2^{8}\right)$. The performance of the proposed technique was improved using an S-random interleaver with $S=15$. The interleaver parses the bits recovered during product coding in the systematic part of the packets. This process improves the overall performance of the scheme and allows faster and more effective correction of the errors. It should be noted that the LDPC decoding is performed only for the packets containing errors and, usually, less than three product decoding iterations are sufficient for the correction of all errors. This is why the additional computational cost with our methodology is very small.

For the experimental evaluation of our methodologies, we report results for a UEP scheme using the optimization algorithm presented in [7] (without determinate bit decoding), an enhanced scheme based on the exploitation of determinate symbols (APC), and a final scheme based on the determinate symbol exploitation and UEP using adaptively-estimated error probabilities (AEC).

In Table 1, the proposed approaches, i.e. APC, AEC and the UEP scheme of [7] for the utilized LDPC codes were compared to the methods in $[1]^{3},[4]$ and [5] for transmission over a BSC chan-

\footnotetext{
${ }^{3}$ The scheme in [1] employes packets of 517 bytes.
} 
Table 1. PSNR comparisons of the proposed schemes with the methods in $[1,5,4]$ for the transmission of "Lenna" for transmission over BSC with $B E R=0.1$.

\begin{tabular}{|c|c|c|c|c|c|c|c|}
\hline $\begin{array}{c}\text { Rate } \\
\text { (in bpp) }\end{array}$ & UEP & APC & AEC & $\begin{array}{c}{[1]} \\
\text { JPEG2000 }\end{array}$ & $\begin{array}{c}{[1]} \\
\text { SPIHT }\end{array}$ & $\begin{array}{c}{[4]} \\
\text { SPIHT }\end{array}$ & {$[5]$} \\
\hline \hline 0.252 & 29.48 & 29.70 & 30.73 & 29.92 & 30.21 & 30.68 & N/A \\
\hline 0.505 & 32.54 & 32.65 & 33.77 & 33.13 & 33.21 & 33.79 & 32.25 \\
\hline 0.994 & 35.80 & 36.00 & 36.80 & 36.03 & 36.10 & N/A & N/A \\
\hline
\end{tabular}

nel with BER equal to 0.1. All the reported mean PSNR values are computed by averaging decoded MSE values and then converting the mean MSE to the corresponding PSNR value. All reported results are averages over 10000 simulations. It can be seen that the AEC approach is significantly better than the APC. The main reason is that the APC algorithm, although it employs the same product coding scheme as the AEC, in general tends to overprotect the source information since it does not take into account the iterative product decoding and its impact on erasure probabilities. The best performing of our schemes, i.e., the AEC approach outperforms the other methods in the comparison apart from the method in [4] with which it has equivalent performance. Specifically, the proposed method outperforms the method in [5] by approximately $1.5 \mathrm{~dB}$ at $0.505 \mathrm{bpp}$. It also achieves better performance than [4] at $0.252 \mathrm{bpp}$ while it is slightly worse at $0.505 \mathrm{bpp}$. However, it is worth noting that the method in [4] uses larger packets (the codewords are as long as the entire transmitted stream). Due to the larger packets, more decoding iterations are required. According to the authors of [4], this fact results in approximately double complexity in comparison to their method in $[1,9]$. It should be noted that both the proposed scheme and the method in [1] use the logarithmic version of SPA. Moreover, the maximum number of SPA iterations in our scheme is 50 while in [1] up to 80 iterations are allowed. Considering the minimal additional computational cost which is introduced by our iterative product decoding (usually only a few packets are needed to be redecoded), we conclude that our scheme and the scheme in [1] are of approximately equal complexity whereas the complexity of our scheme is lower than that of the method in [4]. Finally, the latency problem is less pronounced in our scheme since the decoding of packets that are not erased can start immediately provided that no other packets have been erased previously. In [4], the decoding starts only after the whole bitstream is received.

Table 2. PSNR comparisons of the proposed schemes with the methods in [5] for the transmission of "Lenna" coded at $0.50 \mathrm{bpp}$ over a variety of GE channels.

\begin{tabular}{|c||c|c|c|c|}
\hline GE channel & UEP & APC & AEC & {$[5]$} \\
\hline \hline channel1 & 32.54 & 32.65 & 33.77 & 32.25 \\
\hline channel 2 & 32.54 & 32.65 & 33.77 & 32.22 \\
\hline channel3 & 32.54 & 32.65 & 33.77 & 32.24 \\
\hline channel4 & 32.54 & 32.65 & 33.77 & 32.26 \\
\hline
\end{tabular}

The proposed schemes were also evaluated for transmission over the GE channels used in [5]. Comparisons of our AEC method with the best method in [5], presented in Table 2, demonstrate con- siderable performance improvements of approximately $1.50 \mathrm{~dB}$.

\section{CONCLUSIONS}

We proposed a novel scheme for error resilient image transmission. The proposed scheme is based on a combination of a new technique for the exploitation of determinate symbols in Tanner graph decoding of LDPC codes and a novel product code optimization technique based on error estimation. Experimental evaluation demonstrated the superiority of the proposed system in comparison to recent state-of-the art techniques for image transmission.

\section{REFERENCES}

[1] C. Lan, T. Chu, K. R. Narayanan, and Z. Xiong, "Scalable image and video transmission using irregular repeat-accumulate codes with fast algorithm for optimal unequal error protection.," IEEE Trans. Communications, vol. 52, no. 7, pp. 10921101, Jul. 2004.

[2] H. Jin, A. Khandekar, and R. M. McEliece, "Irregular repeat accumulate codes," in Proc. 2nd Int. Symp. on Turbo Codes, Brest, France, Sep. 2000, pp. 1-8.

[3] B. A. Banister, B. Belzer, and T. R. Fischer, "Robust image transmission using JPEG2000 and turbo-codes," IEEE Signal Processing Letters, vol. 9, no. 4, pp. 117-119, Apr. 2002.

[4] C. Lan, K. R. Elefteriou, and Z. Xiong, "Source optimized irregular repeat accumulate codes with inherent unequal error protection capabilities and their application to image transmission," in Proc. 37th Asilomar Conference on Signals, Pacific Grove, CA, USA, Nov. 2003, vol. 2, pp. 1505-1509.

[5] L. Cao and C. W. Chen, "A novel product coding and recurrent alternate decoding scheme for image transmission over noisy channels," IEEE Trans. Communications, vol. 51, no. 9, pp. 1426-1431, Sep. 2003.

[6] W. E. Ryan, “An Introduction to LDPC Codes," in $C R C$ Handbook for Coding and Signal Processing for Recording Systems (B. Vasic, ed.) CRC Press.

[7] N. Thomos, N. V. Boulgouris, and M. G. Strintzis, "Wireless image transmission using turbo codes and optimal unequal error protection," IEEE Trans. Image Processing, vol. 14, no. 11, pp. 1890-1901, Nov. 2005.

[8] X.-Y. Hu, E. Elefteriou, and D. M. Arnold, "Progressive edgegrowth tanner graphs," in IEEE Global Telecommunications Conference, San Antonio, TX, USA, Nov. 2001, vol. 2, pp. 995-1001.

[9] C. Lan, K. R. Narayanan, and Z. Xiong, "Scalable image and video transmission using irregular repeat-accumulate (ira) codes," in Proc. IEEE Int. Conference on Image Processing, Rochester, NY, USA, Sep. 2002, pp. 717-720. 